International Journal of Pure and Applied Mathematics

Volume 107 No. 2 2016, 381-391

ISSN: 1311-8080 (printed version); ISSN: 1314-3395 (on-line version)

url: http://www.ijpam.eu

doi: 10.12732 /ijpam.v107i2.8

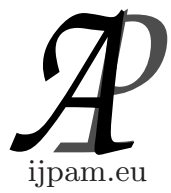

\title{
QUADRATIC FUNCTIONAL EQUATION ON ORTHOGONALITY VECTOR SPACES
}

\author{
Sayed Khalil Ekrami ${ }^{1}$, Madjid Mirzavaziri ${ }^{2}$ \\ ${ }^{1}$ Department of Mathematics \\ Payame Noor University \\ P.O. Box 19395-3697, Tehran, IRAN \\ Department of Pure Mathematics \\ Ferdowsi University of Mashhad \\ P.O. Box 1159, Mashhad 91775, IRAN
}

\begin{abstract}
Let $(X, \perp)$ be a real vector space of dimension at least 3 , with the orthogonality defined on it by:

(i) for all $x \in X, x \perp 0$ and $0 \perp x$,

(ii) for all $x, y \in X \backslash\{0\}, x \perp y$ if and only if $x, y$ are linearly independent.

We show that any orthogonally quadratic mapping on $X$ is a quadratic mapping. Also we prove the Hyers-Ulam stability of orthogonally quadratic functional equation and the HyersUlam stability of orthogonally pexiderized quadratic functional equation.
\end{abstract}

AMS Subject Classification: 39B52, 39B55, 39B82

Key Words: quadratic functional equation, orthogonality space, stability

\section{Introduction and Preliminaries}

As long as we are working in inner product spaces, usually there is no doubt what kind of orthogonality relation we have in mind. Namely, it is the one derived from an inner product and then vectors $x$ and $y$ are orthogonal $(x \perp y)$ if and only if $\langle x, y\rangle=0$.

Received: December 3, 2015

Published: April 9, 2016

${ }^{\S}$ Correspondence author (c) 2016 Academic Publications, Ltd.

url: www.acadpubl.eu 
There are several orthogonality notions on a real normed space such as Birkhoff-James, isosceles, Phythagorean, Roberts and Diminnie (see [10]). J.Rätz in [7] introduced an abstract definition of orthogonality on a real vector space by using four axioms and he investigated the structure of orthogonally additive mappings

$$
f(x+y)=f(x)+f(y) \quad(x \perp y) .
$$

The orthogonally quadratic equation

$$
f(x+y)+f(x-y)=2 f(x)+2 f(y) \quad(x \perp y)
$$

was first investigated by Vajzovic in [12] when $X$ is a Hilbert space, $Y$ is the scalar field, $f$ is continuous and $\perp$ means the Hilbert space orthogonality. Later, Drljević, see [1], generalized this result to $A$-orthogonality on a real or complex Hilbert space defined by $x \perp y$ if and only if $\langle A x, y\rangle=0$, where $A: X \rightarrow X$ is a continuous selfadjoint operator. Fochi in [3] showed that in inner product spaces of dimension not less than 3 , each real valued orthogonally quadratic mapping is unconditionally quadratic. In [4], Fochi proved even more, namely, she showed that both for real and complex valued functions, if $\operatorname{dim} A(X) \geq 3$, then the solutions of the conditional $A$-orthogonal quadratic equation have to be quadratic (unconditionally). Szabó, see [11], generalized the above mentioned results to a symmetric orthogonality introduced by a sesquilinear form on a linear space and for arbitrary mappings with values in an Abelian group.

In [3], Fochi found the general solutions of pexiderized form of orthogonally quadratic equation (1.2), namely

$$
f(x+y)+f(x-y)=2 g(x)+2 h(y) \quad(x \perp y)
$$

in an orthogonality space $X$ in the sense of Rätz.

The stability of functional equation (1.2) was first investigated by Drljević [2] by using $A$-orthogonality on complex Hilbert spaces with the continuity assumption of the function $f$. Sikorska (see [9]) proved the stability of (1.2) on a real Hilbert space and without the continuity assumption of function $f$. Moslehian in [6] proved the stability of the equation (1.3) for functions defined on an orthogonality space. It does not, however, generalize the previous results since it is assumed that $f$ is odd.

One of the open problems in the theory of functional equations is to determine all solutions of the orthogonally quadratic functional equation on an arbitrary orthogonality space, or in a normed space with, e.g., Birkhoff, isosceles or Pythagorean orthogonalities, see [10]. 
In this paper, we show that any orthogonally quadratic function on an orthogonality vector space is a quadratic function. Also we prove the Hyers-Ulam stability of orthogonally quadratic functional equation (1.2) and the HyersUlam stability of orthogonally pexiderized quadratic functional equation (1.3) on orthogonality vector spaces.

\section{The Result}

Let $(X, \perp)$ be a real vector space of dimension at least 3 , with the orthogonality defined on it by

(i) for all $x \in X, x \perp 0$ and $0 \perp x$,

(ii) for all $x, y \in X \backslash\{0\}, x \perp y$ if and only if $x, y$ are linearly independent.

Let $Y$ be a real vector space, then we show that any orthogonally quadratic function $f: X \rightarrow Y$ is a quadratic function.

Lemma 2.1. If $f: X \rightarrow Y$ is a solution of the conditional functional equation (1.2), then $f(2 x)=4 f(x)$ for all $x \in X$.

Proof. Fix $x \in X$, then there are $y, z \in X$ such that $x \perp y, x \perp z$ and $y \perp z$. So by (1.2) we have

$$
\begin{gathered}
f(x+y)+f(x-y)=2 f(x)+2 f(y), \\
f(x+z)+f(x-z)=2 f(x)+2 f(z), \\
f(y+z)+f(y-z)=2 f(y)+2 f(z) .
\end{gathered}
$$

Also since $2 x \perp y+z$ and $2 x \perp y-z$, by (1.2) we get

$$
\begin{aligned}
& f(2 x+y+z)+f(2 x-y-z)=2 f(2 x)+2 f(y+z), \\
& f(2 x+y-z)+f(2 x-y+z)=2 f(2 x)+2 f(y-z) .
\end{aligned}
$$

Similarly applying (1.2) for the pairs of orthogonal vectors $(x+y, x+z),(x+$ $y, x-z),(x+z, x-y)$ and $(x-z, x-y)$ we obtain

$$
\begin{aligned}
& f(2 x+y+z)+f(y-z)=2 f(x+y)+2 f(x+z), \\
& f(2 x+y-z)+f(y+z)=2 f(x+y)+2 f(x-z),
\end{aligned}
$$




$$
\begin{aligned}
& f(2 x-y+z)+f(y+z)=2 f(x+z)+2 f(x-y), \\
& f(2 x-y-z)+f(y-z)=2 f(x-z)+2 f(x-y) .
\end{aligned}
$$

Form (2.4) and (2.5), we get

$$
\begin{aligned}
& f(2 x+y+z)+f(2 x-y-z)+f(2 x+y-z)+f(2 x-y+z) \\
& =4 f(2 x)+2 f(y+z)+2 f(y-z) .
\end{aligned}
$$

Also from (2.6), (2.7),(2.8) and (2.9), we get

$$
\begin{aligned}
& f(2 x+y+z)+f(2 x+y-z)+f(2 x-y+z)+f(2 x-y-z) \\
& =4 f(x+y)+4 f(x-y)+4 f(x+z)+4 f(x-z)-2 f(y+z)-2 f(y-z) .
\end{aligned}
$$

Using the last equations, we have

$$
f(2 x)=f(x+y)+f(x-y)+f(x+z)+f(x-z)-f(y+z)-f(y-z) .
$$

From (2.1), (2.2) and (2.3), we get

$$
f(x+y)+f(x-y)+f(x+z)+f(x-z)-f(y+z)-f(y-z)=4 f(x) .
$$

Therefore by the last two equations, we get $f(2 x)=4 f(x)$ for all $x \in X$ and this completes the proof.

Theorem 2.2. Any solution $f: X \rightarrow Y$ of orthogonally quadratic functional equation (1.2), is a quadratic mapping.

Proof. For any $z \in X$, put $z^{\perp}=\{x \in X, x \perp z\}$ and define the mapping $T_{\mathrm{z}}: z^{\perp} \rightarrow Y$ by

$$
T_{\mathbf{Z}}(x)=f(x+z)-f(x-z),
$$

where $f$ is a solution of equation (1.2). Then $T_{\mathbf{Z}}$ is orthoganally additive on $z^{\perp}$. To see this, let $x, y \in z^{\perp}$ with $x \perp y$, then

$$
\begin{aligned}
T_{\mathbf{z}}(x)+T_{\mathbf{z}}(y) & =f(x+z)-f(x-z)+f(y+z)-f(y-z) \\
& =(f(x+z)+f(y))-(f(x-z)+f(y)) \\
& +(f(y+z)+f(x))-(f(y-z)+f(x)) .
\end{aligned}
$$


Since $(x+z),(x-z) \perp y$ and $(y+z),(y-z) \perp x$, we get

$$
\begin{aligned}
& T_{\mathbf{Z}}(x)+T_{\mathbf{Z}}(y) \\
&= \frac{1}{2}\{(f(x+z+y)+f(x+z-y))-(f(x-z+y)+f(x-z-y)) \\
&+(f(y+z+x)+f(y+z-x))-(f(y-z+x)+f(y-z-x))\} \\
&= f(x+y+z)-f(x+y-z) \\
&= T_{\mathbf{z}}(x+y) .
\end{aligned}
$$

Also since $T_{\mathrm{z}}$ is an odd mapping on $z^{\perp}$, it follows from Theorem 5 of [7] that $T_{\mathbf{Z}}$ is additive on $z^{\perp}$. It follows from (1.2) and that (2.10)

$$
2 f(x+z)=2 f(x)+2 f(z)+T_{\mathbf{Z}}(x) \quad(x \perp z),
$$

and therefore

$$
f(x+z)=f(x)+f(z)+\frac{1}{2} T_{Z}(x) \quad(x \perp z) .
$$

Now we show that $f$ is quadratic on $X$. Let $x, y \in X$ be arbitrary. Then one of the following cases happens for $x$ and $y$ :

First case. Let $x \perp y$. Then it is obvious that $f$ is quadratic.

Second case. Let $x \not \perp y$ and $x+y \perp x-y$. Then replacing $x$ by $x+y$ and $y$ by $x-y$ in (1.2), we have

$$
f(2 x)+f(2 y)=2 f(x+y)+2 f(x-y),
$$

which by Lemma 2.1 implies that $f(x+y)+f(x-y)=2 f(x)+2 f(y)$.

Third case. Let $x \not \perp y$ and $x+y \not \perp x-y$. Then there exits a vector $z \in X$ such that $x \perp z, y \perp z$ and $x+y+z \perp x-y+z$. Replacing $x$ by $x+y+z$ and $y$ by $x-y+z$ in (1.2), we have

$$
f(2(x+z))+f(2 y)=2 f(x+y+z)+2 f(x-y+z),
$$

which implies that

$$
f(x+y+z)+f(x-y+z)=2 f(x+z)+2 f(y) .
$$

It follows from (2.11) that

$$
\begin{aligned}
& f(x+y)+f(z)+\frac{1}{2} T_{\mathbf{Z}}(x+y)+f(x-y)+f(z)+\frac{1}{2} T_{\mathbf{Z}}(x-y) \\
& =2 f(x)+2 f(z)+T_{\mathbf{Z}}(x)+2 f(y) .
\end{aligned}
$$

Since $T_{\mathbf{Z}}$ is additive on $z^{\perp}$, we conclude that $f(x+y)+f(x-y)=2 f(x)+2 f(y)$. This completes the proof. 
In the next theorem, we prove the Hyers-Ulam stability of the orthogonally quadratic functional equation (1.2) on orthogonality vector spaces.

Theorem 2.3. Let $\varepsilon>0$ be a real number and $Y$ be a real Banach space. If $f: X \rightarrow Y$ is a function satisfying the inequality

$$
\|f(x+y)+f(x-y)-2 f(x)-2 f(y)\| \leq \varepsilon
$$

for all orthogonal vectors $x, y \in X$, then there exists a unique quadratic function $q: X \rightarrow Y$ such that

$$
\|f(x)-q(x)\| \leq \frac{3}{2} \varepsilon \quad(x \in X)
$$

Proof. Let $x \in X$. There exist vectors $y, z \in X$ such that $x \perp z, x \perp y$ and $y \perp z$. Since $x+y \perp x-y$, we have

$$
\|f(2 x)+f(2 y)-2 f(x+y)-2 f(x-y)\| \leq \varepsilon .
$$

From (2.12) and (2.14) we get

$$
\|f(2 x)+f(2 y)-4 f(x)-4 f(y)\| \leq 3 \varepsilon
$$

Similarly since $x+z \perp x-z$ and $y+z \perp y-z$, we get

$$
\begin{gathered}
\|f(2 x)+f(2 z)-4 f(x)-4 f(z)\| \leq 3 \varepsilon \\
\|f(2 y)+f(2 z)-4 f(y)-4 f(z)\| \leq 3 \varepsilon
\end{gathered}
$$

Therefore

$$
\begin{aligned}
\|2 f(2 x)-8 f(x)\| & \leq\|f(2 x)+f(2 y)-4 f(x)-4 f(y)\| \\
& +\|f(2 x)+f(2 z)-4 f(x)-4 f(z)\| \\
& +\|f(2 y)+f(2 z)-4 f(y)-4 f(z)\| \leq 9 \varepsilon
\end{aligned}
$$

and then

$$
\|f(2 x)-4 f(x)\| \leq \frac{9}{2} \varepsilon .
$$

An easy induction shows that for an arbitrary positive integer $n$ we have

$$
\left\|f(x)-4^{-\mathrm{n}} f\left(2^{\mathrm{n}} x\right)\right\| \leq \frac{3}{2} \varepsilon\left(1-4^{-\mathrm{n}}\right) \quad(x \in X) .
$$


This implies that for every $x \in X$ the sequence $\left\{4^{-\mathrm{n}} f\left(2^{\mathrm{n}} x\right)\right\}$ is Cauchy in Banach space $Y$. Let $q(x)=\lim _{\mathrm{n} \rightarrow \infty} 4^{-\mathrm{n}} f\left(2^{\mathrm{n}} x\right)$ for each $x \in X$, then relation (2.16) implies that

$$
\|f(x)-q(x)\| \leq \frac{3}{2} \varepsilon \quad(x \in X) .
$$

Choose arbitrarily $x, y \in X$ with $x \perp y$. Then for any $n \in \mathbb{N}, 2^{\mathrm{n}} x \perp 2^{\mathrm{n}} y$, it follows from (2.12) that

$$
\left\|4^{-\mathrm{n}} f\left(2^{\mathrm{n}}(x+y)\right)+4^{-\mathrm{n}} f\left(2^{\mathrm{n}}(x-y)\right)-4^{-\mathrm{n}} f\left(2^{\mathrm{n}} x\right)-4^{-\mathrm{n}} f\left(2^{\mathrm{n}} y\right)\right\| \leq 4^{-\mathrm{n}} \varepsilon .
$$

Letting $n$ tend to infnity we infer that $q(x+y)+q(x-y)=2 q(x)+2 q(y)$ for all $x, y \in X$ with $x \perp y$. From Theorem 2.2, each orthogonally quadratic mapping is quadratic, therefore so is $q$.

To prove the uniqueness, assume $q^{\prime}: X \rightarrow Y$ to be another quadratic mapping such that $\left\|f(x)-q^{\prime}(x)\right\| \leq \frac{3}{2} \varepsilon$ for all $x \in X$. Then, for each $x \in X$ and all $n \in \mathbb{N}$ it follows from Lemma 2.1 that

$$
\left\|q(x)-q^{\prime}(x)\right\| \leq 4^{-\mathrm{n}}\left\|\left(q\left(2^{\mathrm{n}} x\right)-f\left(2^{\mathrm{n}} x\right)\right)+\left(f\left(2^{\mathrm{n}} x\right)-q^{\prime}\left(2^{\mathrm{n}} x\right)\right)\right\| \leq 4^{-\mathrm{n}}(3 \varepsilon)
$$

which implies $q=q^{\prime}$ and finishes the proof.

Lemma 2.4. Let $\varepsilon>0$ be a real number and $Y$ be a real Banach space. If $f: X \rightarrow Y$ is an odd function satisfying the inequality

$$
\|f(x+y)+f(x-y)-2 f(x)\| \leq \varepsilon
$$

for all orthogonal vectors $x, y \in X$, then there exists a unique additve function $a: X \rightarrow Y$ such that

$$
\|f(x)-a(x)\| \leq 2 \varepsilon \quad(x \in X) .
$$

Proof. Interchanging $x$ by $y$ in (2.17) we get

$$
\|f(x+y)-f(x-y)-2 f(y)\| \leq \varepsilon .
$$

Using (2.17) and (2.19) we get

$$
\|f(x+y)-f(x)-f(y)\| \leq \varepsilon .
$$

Since $x+y \perp x-y$, from the above inequality we get

$$
\|f(2 x)-f(x+y)-f(x-y)\| \leq \varepsilon .
$$


From (2.17) and (2.21) we have

$$
\left\|f(x)-2^{-1} f(2 x)\right\| \leq \varepsilon .
$$

An easy induction shows that for an arbitrary positive integer $n$ we have

$$
\left\|f(x)-2^{-\mathrm{n}} f\left(2^{\mathrm{n}} x\right)\right\| \leq 2 \varepsilon\left(1-2^{-\mathrm{n}}\right) \quad(x \in X) .
$$

This implies that for every $x \in X$ the sequence $\left\{2^{-\mathrm{n}} f\left(2^{\mathrm{n}} x\right)\right\}$ is Cauchy in Banach space $Y$. Let $a(x)=\lim _{\mathrm{n} \rightarrow \infty} 2^{-\mathrm{n}} f\left(2^{\mathrm{n}} x\right)$ for each $x \in X$, then relation (2.22) implies that

$$
\|f(x)-a(x)\| \leq 2 \varepsilon \quad(x \in X) .
$$

Choose arbitrarily $x, y \in X$ with $x \perp y$. Then for any $n \in \mathbb{N}, 2^{\mathrm{n}} x \perp 2^{\mathrm{n}} y$, it follows from (2.20) that

$$
\left\|2^{-\mathrm{n}} f\left(2^{\mathrm{n}}(x+y)\right)-2^{-\mathrm{n}} f\left(2^{\mathrm{n}} x\right)-2^{-\mathrm{n}} f\left(2^{\mathrm{n}} y\right)\right\| \leq 2^{-\mathrm{n}} \varepsilon .
$$

Letting $n$ tend to infnity we infer that $a(x+y)=a(x)+a(y)$ for all $x, y \in X$ with $x \perp y$.

Finally, on account of Theorem 5 of [7], each odd orthogonally additive mapping is additive, therefore so is $a$.

To prove the uniqueness, assume $a^{\prime}: X \rightarrow Y$ to be another additive mapping such that $\left\|f(x)-a^{\prime}(x)\right\| \leq 2 \varepsilon$ for all $x \in X$. Then, for each $x \in X$ and all $n \in \mathbb{N}$ one has

$$
\left\|a(x)-a^{\prime}(x)\right\| \leq n^{-1}\left\|(a(n x)-f(n x))+\left(f(n x)-a^{\prime}(n x)\right)\right\| \leq 4 n^{-1} \varepsilon
$$

which implies $a=a^{\prime}$ and finishes the proof.

In the next theorem, we prove the Hyres-Ulam stability of orthogonally pexiderized quadratic functional equation (1.3) on orthogonality vector spaces.

Theorem 2.5. Let $\varepsilon>0$ be a real number and $Y$ be a real Banach space. If $f, g, h: X \rightarrow Y$ are fucntions satisfying the inequality

$$
\|f(x+y)+f(x-y)-2 g(x)-2 h(y)\| \leq \varepsilon
$$

for all orthogonal vectors $x, y \in X$, then there exists a unique additive mapping $a: X \rightarrow Y$ and a unique quadratic mapping $q: X \rightarrow Y$ such that

$$
\left\{\begin{array}{l}
\|f(x)-a(x)-q(x)-f(0)\| \leq 14 \varepsilon+14\|h(0)\| \\
\|g(x)-a(x)-q(x)-g(0)\| \leq 15 \varepsilon+16\|h(0)\| \\
\|h(x)-q(x)\| \leq 7 \varepsilon+7\|h(0)\|
\end{array}\right.
$$

for all $x \in X$. 
Proof. Putting $y=0$ in $(2.23)$ we have

$$
\|f(x)-g(x)\| \leq \frac{1}{2} \varepsilon+\|h(0)\| \quad(x \in X) .
$$

Suppose that $x, y \in X$ be two orthogonal vectors. Using the above inequality and (2.23) we have

$$
\|f(x+y)+f(x-y)-2 f(x)-2 h(y)\| \leq 2 \varepsilon+2\|h(0)\| .
$$

Putting $x=0$ in $(2.25)$ we get

$$
\|f(y)+f(-y)-2 f(0)-2 h(y)\| \leq 2 \varepsilon+2\|h(0)\|
$$

It follows from (2.25) and (2.26) that

$$
\|f(x+y)+f(x-y)-2 f(x)-f(y)-f(-y)+2 f(0)\| \leq 4 \varepsilon+4\|h(0)\| .
$$

Define the function $f_{1}: X \rightarrow Y$ by

$$
f_{1}(x)=\frac{1}{2}(f(x)+f(-x))-f(0) \quad(x \in X) .
$$

Then $f_{1}$ is an even function and by $(2.27)$ we have

$$
\left\|f_{1}(x+y)+f_{1}(x-y)-2 f_{1}(x)-2 f_{1}(y)\right\| \leq 4 \varepsilon+4\|h(0)\| .
$$

By Theorem 2.3 there exists a unique quadratic mapping $q: X \rightarrow Y$ such that

$$
\left\|f_{1}(x)-q(x)\right\| \leq 6 \varepsilon+6\|h(0)\| \quad(x \in X) .
$$

Replacing $x$ by $-x$ in $(2.27)$, we have

$$
\|f(-x+y)+f(-x-y)-2 f(-x)-f(y)-f(-y)+2 f(0)\| \leq 4 \varepsilon+4\|h(0)\| .
$$

From (2.27) amd (2.29) we gat

$$
\begin{array}{r}
\|f(x+y)+f(x-y)-f(-x+y)-f(-x-y)-2(f(x)-f(-x))\| \\
\leq 8 \varepsilon+8\|h(0)\| .
\end{array}
$$

Define the function $f_{2}: X \rightarrow Y$ by

$$
f_{2}(x)=\frac{1}{2}(f(x)-f(-x)) \quad(x \in X) .
$$


Then $f_{2}$ is an odd mapping and by (2.30) we have

$$
\left\|f_{2}(x+y)+f_{2}(x-y)-2 f_{2}(x)\right\| \leq 4 \varepsilon+4\|h(0)\| .
$$

By Lemma 2.4 there exists a unique additive mapping $a: X \rightarrow Y$ such that

$$
\left\|f_{2}(x)-a(x)\right\| \leq 8 \varepsilon+8\|h(0)\| \quad(x \in X) .
$$

Using (2.28) and (2.32) we obtain

$$
\|f(x)-a(x)-q(x)-f(0)\| \leq 14 \varepsilon+14\|h(0)\| \quad(x \in X) .
$$

It follows from the last inequality and (2.24) that

$$
\|g(x)-a(x)-q(x)-g(0)\| \leq 15 \varepsilon+16\|h(0)\| \quad(x \in X) .
$$

Also using (2.26) and (2.28) we get

$$
\|h(x)-q(x)\| \leq 7 \varepsilon+7\|h(0)\| \quad(x \in X) .
$$

\section{References}

[1] F. Drljevic̀, On a functional which is quadratic on A-orthogonal vectors, Publ. Inst. Math. (Beograd) 54 (1986), 63-71.

[2] F. Drljevic̀, On the stability of the functional quadratic on A-orthogonal vectors, Publ. Inst. Math. (Beograd) (N.S.) 36 (50), (1984), 111-118.

[3] M. Fochi, Alcune equazioni funzionali condizionate sui vettori ortogonali, Rend. Sem. Mat. Univ. Politec. Torino 44, (1986) 397-406.

[4] M. Fochi, Functional equations in A-orthogonal vectors, Aequationes Math. 38 (1989), $28-40$.

[5] S. Gudder, D. Strawther, Orthogonally additive and orthogonally increasing functions on vector spaces, Pacific J. Math. 58 (1975), 427-436.

[6] M.S. Moslehian, On the orthogonal stability of the Pexiderized quadratic equation, $J$. Differ. Equ. Appl. 11, (2005), 999-1004.

[7] J. Rätz, On orthogonally additive mappings, Aequationes Math. 28 (1985), 35-49. 
[8] J. Rätz, Gy. Szabó, On orthogonally additive mappings IV, Aequationes Math. 38 (1989), 73-85.

[9] J. Sikorska, Generalized orthogonal stability of some functional equations, J. Inequal. Appl. (2006), Art. ID 12404.

[10] J. Sikorska, Orthogonalities and functional equations, Aequationes Math. 892 (2015), 215-277.

[11] Gy. Szabó, Sesquilinear-orthogonally quadratic mappings, Aequationes Math. 40 (1990), 190-200.

[12] F. Vajzović, Über das Funktional H mit der Eigenschaft: $(x, y)=0 \Rightarrow H(x+y)+H(x-$ $y)=2 H(x)+2 H(y)$, Glasnik Mat. Ser. III 2 (22) (1967), 73-81. 
